\title{
Caracterização do estresse nos enfermeiros de unidades de terapia intensiva
}

\author{
CHARACTERIZATION OF STRESS IN INTENSIVE CARE UNIT NURSES \\ CARACTERIZACIÓN DEL ESTRÉS EN LAS ENFERMERAS DE UNIDADES \\ DE CUIDADOS INTENSIVOS
}

\author{
Francine Jomara Lopes Guerrer ${ }^{1}$, Estela Regina Ferraz Bianchi
}

\begin{abstract}
RESUMO
O estudo propõe uma caracterização dos enfermeiros que atuam em unidades de terapia intensiva (UTIS) das Regiões Brasileiras e a associação do nível de estresse relatado com idade, cargo ocupado, tempo de formado e freqüência a cursos de pósgraduação. Os dados foram coletados utilizando-se a Escala Bianchi de Stress, constituída por caracterização sociodemográfica e 51 itens das atividades desempenhadas por enfermeiros. A amostra foi composta por 263 enfermeiros, sendo feminina (91,6\%), jovem ( $80,2 \%<40$ anos), entre 2 e 5 anos de formado (34,6\%), 87,8\% atuando como enfermeiros assistenciais, $74,5 \%$ com pós-graduação lato sensu. Os enfermeiros obtiveram nível de estresse entre médio e alerta $(60,1 \%)$. Houve associação estatisticamente significante $(p \varangle 0,05)$ entre domínios C (administração de pessoal) e $D$ (assistência de enfermagem) e realização de curso de pós-graduação. Concluindo, tanto os enfermeiros como hospitais devem investir esforços para obter subsídios para a prestação de assistência e estratégias de enfrentamento do estresse.
\end{abstract}

\section{DESCRITORES}

Estresse.

Unidades de Terapia Intensiva.

Enfermagem.

\begin{abstract}
This study proposes a characterization of the nurses who work in intensive care units (ICU) in different regions of Brazil and the association of the stress level they report with age, position, time since graduation, and enrollment in Graduate courses. The data were collected using the Bianchi Stress Inventory, consisting of socio-demographic information and 51 items of the activities nurses perform. The sample was comprised of 263 nurses, most of them female (91.6\%); young ( $80.2 \%$ are less than 40-years old); graduated between 2 and 5 years previously $(34.6 \%)$; with latu sensu Graduate studies (74.5\%). Most individual stress scores varied from medium to alert $(60.1 \%)$. There was statistically significant association $(p<0.05)$ between areas $C$ (administration of personnel) and $D$ (nursing assistance). In conclusion, both nurses and hospitals must make efforts to get subsidies for improving assistance conditions and for strategies to cope with stress.
\end{abstract}

\section{KEY WORDS}

Stress.

Intensive Care Units.

Nursing.

\section{RESUMEN}

En este estudio se propone la caracterización de los enfermeros que trabajan en unidades de cuidados intensivos (UCls) de las Regiones Brasileñas y la asociación del nivel de estrés con la edad, cargo ocupado, tiempo de egresado y la frecuencia a cursos de postgrado. Los datos fueron recolectados utilizándose la Escala Bianchi de Estrés, constituida por caracterización sociodemográfica y 51 items de las actividades desempeñadas por enfermeros. La muestra estuvo conformada por 263 enfermeros, siendo del sexo femenino (91,6\%), joven (80,2\%<40 años), entre 2 y 5 años de egresado (34,6\%), 87,8\% trabajando como enfermeros asistenciales, $74,5 \%$ con postgrado latosensu. Los enfermeros obtuvieron un nivel de estrés entre medio y alerta $(60,1 \%)$. Hubo asociación estadísticamente significativa $(p \varangle 0,05)$ entre dominios $C$ (administración de personal) y $D$ (asistencia de enfermería) y realización del curso de postgrado. Concluyendo, tanto los enfermeros como los hospitales deben invertir esfuerzos para obtener subsidios para la prestación de asistencia y estrategias de enfrentamiento del estrés.

\section{DESCRIPTORES}

Estrés.

Unidades de terapia Intensiva.

Enfermería.

${ }^{1}$ Enfermeira do Hospital Sírio Libanês, Especialista em Enfermagem Cardiovascular e Mestre pela Escola de Enfermagem USP. São Paulo, SP, Brasil. fran.jl@terra.com.br ${ }^{2}$ Enfermeira. Professora Associada da Escola de Enfermagem da USP. São Paulo, SP, Brasil. erfbianc@usp.br 


\section{INTRODUÇÃO}

Estresse é um problema atual, estudado por vários profissionais, pois apresenta risco para o equilíbrio normal do ser humano. Há cada vez mais uma preocupação com a saúde dos trabalhadores para que os danos sejam evitados e segundo a Organização M undial de Saúde (OMS) há um favorecimento da saúde física e mental quando o trabalho se adapta às condições do trabaIhador e quando os riscos para a sua saúde estão sob controle ${ }^{(1)}$.

A primeira definição de estresse foi dada em 1956, definindo-o como uma reação inespecífica do corpo a qualquer demanda, interna ou externa. 0 autor enfatiza que o estresse é uma parte normal do funcionamento do corpo, sendo uma conseqüência do ato de viver ${ }^{(2)}$.

Outros autores conceituam o estresse como um estado em que ocorre um desgaste anormal do corpo humano e/ ou uma diminuição da capacidade de trabalho ocasionado basicamente por uma incapacidade prolongada de 0 indivíduo tolerar, superar ou se adaptar às exigências de natureza psíquica existentes no seu ambiente de vida. É observado em todas as faixas etárias e geralmente está relacionado ao estilo de vida do indivíduo ${ }^{(3-4)}$.

0 modelo interacionista define estresse como qualquer estímulo que demande do ambiente externo ou interno e que taxe ou exceda as fontes de adaptação de um indivíduo ou sistema social, com um fator determinante da severidade do estressor ${ }^{(5)}$. Atualmente, é o modelo mais divulgado entre os estudiosos de estresse, por interagir 0 ambi-

A primeira autora que designou a profissão enfermagem, como estressante, relacionou o estresse ao trabalho com pessoas doentes que requerem grande demanda de compaixão, sofrimento e empatia. ente e a pessoa ou o grupo, como responsáveis e atuantes no processo.

A primeira autora que designou a profissão, enfermagem, como estressante, relacionou o estresse ao trabalho com pessoas doentes que requerem grande demanda de compaixão, sofrimento e simpatia(6). 0 enfermeiro, lidando com essa situação pode se sentir irritado, deprimido, desapontado e esses sentimentos são considerados incompatíveis com o desempenho profissional, trazendo conseqüentemente a culpa e o aumento da ansiedade.

Ser responsável por pessoas, como no caso dos enfermeiros, obriga a um maior tempo de trabalho dedicado à interação, aumentando a probabilidade de ocorrência do estresse por conflitos interpessoais( ${ }^{(7)}$.

0 trabalho do enfermeiro, inserido nas instituições de saúde, é muitas vezes multifacetado, dividido e submetido a uma diversidade de cargos que são geradores de desgaste. Em contrapartida, o trabalho também se constitui em fonte de prazer e satisfação, que são potencializadoras das capacidades humanas, na promoção de saúde e vida ${ }^{(8)}$.
A UTI é uma unidade onde se encontram internados pacientes que necessitam de cuidados diretos e intensivos, pois seu quadro de saúde pode facilmente evoluir para a morte; além disso, é considerado um setor fechado onde 0 entrosamento com outros setores é bastante diminuído ${ }^{(9)}$.

A assistência prestada à pacientes em UTI é bastante polêmica, se de um lado ela requer intervenções rápidas, de outro, não se tem dúvida de que são espaços naturalmente mobilizadores de emoções e sentimentos que freqüentemente se expressam de forma muito intensa. Ser enfermeiro na UTI envolve a realização de um trabalho permeado por ambigüidades, aspectos gratificantes e limitantes que estão presentes no seu mundo e na vida ${ }^{(10)}$.

O estudo sobre a atuação do enfermeiro quanto ao estresse é atual e necessário. Esse estudo tem como objetivos caracterizar a população de enfermeiros que atuam em UTI no Brasil e associar o nível de estresse relatado pelos enfermeiros com idade, cargo ocupado, tempo de formado e freqüência a cursos de pós-graduação.

\section{MÉTODO}

A amostra foi constituída por 263 enfermeiros atuantes nas UTIs dos diversos hospitais de alta complexidade das capitais dos estados brasileiros. Essa amostra foi constituída pelos enfermeiros de UTI que responderam à pesquisa realizada. 0 critério de inclusão foi trabalhar em UTI de um hospital de alta complexidade das capitais do Brasil.

Como primeira etapa, foi enviada solicitação para 409 hospitais que estavam englobados como prestadores de alta complexidade no registro do DATASUS ${ }^{(11)}$ em dezembro de 2004. Desse total, 81 hospitais $(19,8 \%)$ aceitaram participar da pesquisa e pertenciam às diferentes capitais brasileiras, com exceção dos estados de Santa Catarina, Roraima e Rondônia. Desses 81 hospitais, os enfermeiros assinalaram no questionário o local de trabalho e para o presente estudo, foram analisadas as respostas somente dos enfermeiros de UTI.

0 projeto foi submetido e aprovado pela Comissão de Ética em Pesquisa da Escola de Enfermagem da USP (Processo n. 228/2003). Cada enfermeiro das instituições participantes recebeu um envelope contendo o questionário com a carta convite e o termo de responsabilidade, oferecendo detalhes e reiterando o sigilo das informações obtidas, 0 anonimato e a participação livre e espontânea na pesquisa, e o envelope pré-franquiado para o envio das respostas. 0 período de coleta dos dados foi de maio a dezembro de 2005

Os dados foram coletados utilizando-se a Escala Bianchi de Stress, constituída por dados de caracterização sócio-demográfica e 51 itens que versam sobre as 
atividades desempenhadas pelos enfermeiros. Com a finalidade de promover a comparação e estudo dos dados, foram determinados os escores de estresse em 6 domínios englobando 0 relacionamento (A): itens 40 a 46, 50 e 51; funcionamento da unidade (B): 1 a 6; administração de pessoal (C): 7 a 9 e 12 a 14; assistência de enfermagem (D): 16 a 30; coordenação da unidade (E): 10, 11, 15, 31, 32, 38, 39 e 47 e condições de trabalho (F): 33 a 37, 48 e 49.

Cada item foi assinalado, tendo como classificação 0 , para atividades não realizadas; 1 como "pouco estresse", 4 como nível "médio" e 7 como "altamente estressante".

Para cada enfermeiro foi calculado o nível de estresse, realizando-se a média real isto é, a somatória dos itens, dividindo-se pelos itens assinalados e subtraindo-se 0 número de zeros marcados, para que houvesse a média que demonstrasse 0 nível de estresse para as atividades realizadas pelos enfermeiros, e não havendo a interferência de itens não realizados.

Para cada domínio, também foi realizada a média real. Foi calculada a somatória dos itens componentes de cada domínio e dividido pelo número de respondentes que assinalaram os itens com valores de 1 a 7 . Também foi calculada a média real para cada item isoladamente.

Os níveis obtidos foram classificados em baixo (até 3,0), médio (de 3,1 a 4,0), alerta (de 4,1 a 5,9) e alto (acima de 6,0).

A análise estatística foi descritiva e inferencial, utilizando 0 alfa de Cronbach para avaliar a confiabilidade dos dados, os testes não-paramétricos de $M$ ann-Whitney e Kruskall-Wallis para testar a diferença nas classificações de grupos independentes. 0 alfa de Cronbach da escala total para este estudo foi de 0,8366 , considerado satisfatório.

\section{RESULTADOS E DISCUSSÃO}

Houve um predomínio do sexo feminino com 241 $(91,6 \%)$ do total da população, coincidindo com o perfil de enfermeiros em geral do Brasil, onde há um predomínio do sexo feminino. Os indicadores e dados básicos do Brasil de 2004 corroboram com a descrição desse perfil de enfermeiros, pois é descrito que aproximadamente $92 \%$ do total de enfermeiros são do sexo feminino(11).

0 trabalho de enfermagem é marcante não somente por caracterizar-se como uma profissão essencialmente integrada por pessoas do sexo feminino, como também pela especificidade das ações desenvolvidas no dia-adia. As profissionais de enfermagem convivem com a dinâmica das organizações no desenvolvimento de suas atividades, ao mesmo tempo em que gerenciam suas vidas como pessoas, esposas e mães ${ }^{(12)}$. Essa situação, de desenvolver múltiplas atividades, com vínculos de trabaIho formais ou não, pode também gerar estresse já que essas mulheres além de trabalharem fora do convívio fa- miliar pensam em seus filhos e se preocupam com os cuidados domiciliares. No entanto, em outro estudo relacionado ao estresse de enfermeiras, mostrou que as atividades relacionadas à vida pessoal, tais como responsabilidades com a casa, com os filhos e outras atividades domésticas ao invés de estressantes, podem funcionar como suporte emocional(13).

Quanto ao nível individual de estresse obteve-se : 105 $(39,9 \%)$ com baixo nível de estresse, 96 (36,5\%) com médio nível de estresse, $62(23,6 \%)$ em alerta para alto nível de estresse e nenhum com alto nível de estresse. A maioria desses enfermeiros $(60,1 \%)$ ficou entre nível médio e alerta para estresse.

$\mathrm{Na}$ análise do nível de estresse para cada domínio, o que apresentou o maior nível de estresse foi o C (administração de pessoal) com escore de 3,99; seguido do $F$ (condições de trabalho) com escore de 3,97; E (coordenação das atividades) com escore de 3,83; $D$ (assistência de enfermagem) com escore de 3,62; $B$ (funcionamento da unidade) com escore de 3,45; A (relacionamento) com escore de 3,29.

Diante disso, pode-se afirmar que os enfermeiros desse estudo apresentaram maiores índices de estresse nas atividades relacionadas à administração de pessoal e nas condições de trabalho para desempenho do enfermeiro, considerado de nível médio, pois permaneceu abaixo de 4,0 .

$\mathrm{Na}$ análise da interferência do sexo na ocorrência de estresse, o sexo masculino apresentou índices mais elevados de estresse nos domínios $A, D$, e F, em que se encontram atividades relacionadas ao relacionamento interpessoal (A) com média de 3,35, aos cuidados de enfermagem (D) com média de 3,74 e condições de trabalho (F) com média de 4,03. Quando verificado a média total para cada sexo, o masculino apresentou níveis mais elevados de estresse, 3,68. As diferenças obtidas entre os dois sexos não foram estatisticamente significantes.

Quanto à faixa etária, a população estudada foi considerada jovem, (80,2\% com menos de 40 anos), que é 0 perfil de enfermeiros esperado para esse setor, pois, mesmo durante a graduação, são motivados à prestação de assistência a pacientes críticos, além de serem pacientes que requerem maior tempo de cuidado. Essa situação também foi encontrada em outra pesquisa com enfermeiros, onde $68,8 \%$ dos participantes tinham menos de 40 anos de idade ${ }^{(14)}$.

Como justificativa para a considerável redução de enfermeiros com mais de 40 anos, pode-se citar a possibilidade desses enfermeiros estarem em cargos administrativos ou outras áreas como a de ensino, já que têm experiência na profissão.

Ao comparar os domínios e faixa etária, verificou-se que para a faixa etária de 31 a 40 anos, as médias de escore de estresse foram maiores nos domínios D (assis- 
tência de enfermagem), e $E$ (coordenação da unidade), sendo respectivamente 3,63 e 4,0. A faixa etária, entre 41 e 50 anos, ficou com o maior escore, 4,04 no domínio C (administração de pessoal) e a faixa com mais de 51 anos, nos domínios A (relacionamento) com 3,72, no B (funcionamento da unidade) com 4,35 e no $F$ (condições de trabaIho) com 4,33. A faixa etária entre 31 e 40 anos foi a que apresentou níveis mais elevados de estresse, entretanto, não foi observada diferença estatisticamente significante.

Ao se verificar a média total para cada faixa de idade, os enfermeiros com idade entre 31 e 40 anos obtiveram escore de 3,75, os com 41 a 50 anos de 3,68 e os com mais de 51 anos, com 3,63. Esses resultados diferem dos descritos na literatura, demonstrando que os enfermeiros de UTI têm índices de estresse semelhantes, provavelmente pelo tipo de trabalho ali realizado. Para se comprovar essa questão, é necessário planejar um estudo tendo como foco principal o estudo da interferência da idade no nível de estresse do enfermeiro de UTI.

Quanto ao cargo ocupado, os dados demonstram um predomínio de enfermeiros que atuam na assistência 231 (87,8\%), já que na UTI o enfermeiro é responsável pela assistência direta ao paciente. 0 enfermeiro em UTI deve ter base de conhecimento que facilite a capacidade de perceber uma grande variedade de questões, bem como informações altamente definidas e específicas. ${ }^{(15)}$ Para tanto, o enfermeiro deve prestar a assistência à beira do leito desse paciente para identificar possíveis alterações o mais rápido possível.

Comparando os domínios e o cargo em que os enfermeiros ocupam, o cargo assistencial apresentou índices mais elevados de estresse para os domínios D (assistência de enfermagem) e E (coordenação da unidade), sendo respectivamente 3,64 e 3,84. Os cargos de chefe/ coordenador/líder apresentaram maiores índices de estresse para os domínios A (relacionamento), B (funcionamento da unidade) e $C$ (administração de pessoal), respectivamente $3,57,4,13$ e 4,19. Os cargos de diretor/supervisor/gerente apresentaram índices mais elevados para o domínio $F$ (condições de trabalho), sendo 4,31. Ao verificar a média total de estresse para cada cargo, tem-se que os enfermeiros chefe/coordenador/líder apresentaram níveis mais elevados, com média de 3,78.

Os resultados do presente estudo são semelhantes aos encontrados em pesquisa realizada sobre o estresse ocupacional de Enfermeiros, onde foram identificados os estressores para os enfermeiros assistenciais como: recursos inadequados, atendimento ao paciente, relações interpessoais e carga emocional e para os enfermeiros administrativos foram levantados como recursos inadequados, relacionados à assistência: relações interpessoais, cobranças, sobrecarga de trabalho, reconhecimento profissional e poder de decisão(13).

Pode-se notar que o nível do estresse entre os cargos e domínios, está relacionado geralmente ao tipo de traba-
Iho que esses enfermeiros exercem, ou seja, os enfermeiros chefes apresentaram níveis mais elevados em domínios com atividades administrativas, assim como os enfermeiros assistenciais apresentaram índices mais elevados de estresse para domínios mais relacionados à assistência do paciente. Esse dado leva à consideração de que as atividades inerentes ao cargo são consideradas estressantes, e a instituição hospitalar, como a de ensino, necessitam de estratégias para conscientizar os enfermeiros de que a situação profissional precisa de investimento individual e organizacional para melhor adaptação e menor efeito adverso para a vivência profissional.

Quando comparado o tempo de formado com os domínios, observou-se que os enfermeiros com menos de 1 ano e de 2 a 5 anos de formados não apresentaram níveis mais elevados de estresse para nenhum domínio. Os enfermeiros com 6 a 10 anos apresentaram níveis mais elevados para os domínios A (relacionamento) e C (administração de pessoal), respectivamente 3,40 e 4,11. Para enfermeiros com 11 a 15 anos apresentaram níveis mais elevados para os domínios A (relacionamento), D (assistência de enfermagem), $E$ (coordenação da unidade) e $F$ (condições de trabalho), sendo os níveis de 3,40, 3,76, 3,97 e 4,09. Para mais de 16 anos, os níveis foram mais elevados no domínio $B$ (funcionamento da unidade) com 3,79. Ao se verificar a média total para cada faixa de tempo de formado, os enfermeiros com 11 a 15 anos de formado apresentaram níveis mais elevados de estresse, com média de 3,79, ao contrário do esperado, que quanto maior o tempo de formado, menor o estresse, que seria explicado pelo fato do enfermeiro apresentar maior segurança técnica e controle sobre as situações que surgem em UTI, de tal forma que estas não se configurariam como estressantes.

A maioria dos enfermeiros desse estudo tem pelo menos um curso de pós-graduação, sendo $74,5 \%$ do total, uma característica que cada vez mais está sendo observada entre os enfermeiros jovens, que se colocam no mercado de trabalho com a capacitação de especializações, principalmente para atuação em unidades de prestação de assistência complexa, como ocorre em UTIs. A enfermagem em UTI requer uma capacidade de lidar com situações cruciais com uma velocidade e precisão geralmente não necessárias em outras unidades assistenciais. Requer competência na integração de informação, construção de julgamentos e estabelecimento de prioridades, porque, quando o distúrbio acomete um sistema orgânico, outros sistemas são envolvidos na tentativa de adaptar-se ao desequilíbrio ${ }^{(16)}$. Para adquirir esse tipo de habilidade esses enfermeiros procuram se especializar.

A freqüência dos enfermeiros que atuam em UTI em cursos de pós-graduação tem como conseqüência dois pontos positivos. 0 primeiro é o estímulo oferecido por esses cursos na elaboração de pesquisas, geralmente derivadas de questões da vivência prática; segundo, por atuarem na área assistencial, a possibilidade de implementação dos resultados obtidos é maior ${ }^{(16)}$. 
Foi realizado um levantamento de enfermeiros de diferentes instituições de ensino, assistência e pesquisa quanto à contratação de recém-formados para trabalhar em UTI. Dentre as 9 pesquisadas, 4 delas responderam que contratavam, porém somente após, pelo menos, 3 meses de treinamento, e não assumindo algumas áreas críticas como cirurgia cardíaca. As que não contratavam justificavam dizendo que preferiam enfermeiros mais experientes e não apenas com conhecimento teórico ${ }^{(17)}$.

0 resultado desse levantamento pode justificar o motivo que na amostra deste estudo há um número elevado de enfermeiros com pelo menos uma pós-graduação, e número pequeno de enfermeiro com menos de um ano de formado 20 (7,6\%). Enfermeiros sem especialização não são muito aceitos em UTI, por ser um setor de cuidados críticos e a especialização dá ao profissional mais experiência profissional.

Outro fator que deve ser analisado é o surgimento das sociedades de enfermeiros especialistas, em especial a de enfermeiros de UTI. Com o advindo das sociedades, surge também o oferecimento da possibilidade de obter 0 título de especialista e em paralelo a procura por cursos de especialização na área ou em áreas correlatas.

Quanto à presença de pós-graduação e a análise do nível de estresse desses enfermeiros, os que tinham uma pós-graduação apresentaram índices elevados de estresse nos domínios C (administração de pessoal), D (assistência de enfermagem), E (coordenação da unidade) e F (condições de trabalho), sendo estatisticamente significante para os domínios $C(p=0,028)$ e $D(p=0,043)$, ou seja, observa-se nos dois casos que os Enfermeiros com curso de pós-graduação apresentaram maiores níveis de estresse.

Ao analisar a média total para a presença ou não de pós-graduação, foram obtidos níveis mais elevados de estresse para os que tinham pós-graduação, sendo o nível de 3,71 .

A freqüência a cursos de pós-graduação poderia resultar em diminuição da ocorrência de estressores, como observado em outra pesquisa, porém esse resultado não foi observado no presente estudo ${ }^{(18)}$.

Pode-se analisar que a freqüência a um curso de pósgraduação, ou a obtenção do título de especialista em concursos de sociedades, que antes poderia ser um diferencial na obtenção de uma colocação no mercado de trabalho, cada vez mais está sendo avaliado como um requisito para assunção de determinados cargos. Em contrapartida, também pode acontecer que o enfermeiro preparado, com diversos cursos, não obtenha o reconhecimento esperado e que ocorra uma insatisfação com as atividades desempenhadas que podem estar aquém de seu preparo educacional. Ou ainda, a procura por diversas especializações devido à sua falta de adaptação às condições do trabalho realizado. Para analisar este tema sugere-se a realização de trabalho detalhado, avaliando a satisfação no trabalho e a freqüência a cursos de pósgraduação.

Contudo fica patente que a resposta aos estressores da UTI depende da avaliação individual e que a administração de pessoal é um estressor presente na atuação do enfermeiro em UTI. Tanto a instituição hospitalar deve investir em recursos humanos e em subsídios para a prestação de assistência que está cada vez mais comple$x a$, como o próprio enfermeiro deve conhecer seus limites e procurar estratégias para enfrentar os estressores vividos.

\section{CONCLUSÕES}

Neste estudo o perfil demográfico da população foi hegemonicamente do sexo feminino $(91,6 \%)$, população mais jovem, sendo com menos de 40 anos de idade $(80,2 \%)$, enfermeiros com cargo assistencial (87,8\%), com pós-graduação $(74,5 \%)$.

Obteve-se significância estatística em relação à presença de pós-graduação para o domínio $C$ e $D$, os enfermeiros que tinham algum tipo de pós-graduação apresentaram maiores níveis de estresse.

Não se pode afirmar que as características da população estudada são determinantes para a ocorrência de estresse entre os enfermeiros de UTI, entretanto, fica patente que há necessidade de se instrumentalizar cada vez mais o enfermeiro para que a avaliação do estressor seja feita com base nos mecanismos de enfrentamento disponíveis, possibilitando a menor ocorrência de estresse para o indivíduo.

0 enfermeiro e a instituição hospitalar devem reconhecer os estressores que estão presentes no trabalho e procurar mecanismos e estratégias de enfrentamento individual e grupal para diminuir a ocorrência de estresse profissional.

\section{REFERÊNCIAS}

1. Carvalho DV, Lima FCA, Costa TMPF, Lima EDRP. Enfermagem em setor fechado: estresse ocupacional. REME Rev $\mathrm{M}$ in Enferm. 2004;8(2):290-4.

2. Selye H. The stress of life. New York: McGraw-Hill; 1956.
3. Alves GLB. Stress: diagnóstico e tratamento. Curitiba: RELISUL; 1992.

4. Couto HA. Stress e qualidade de vida do executivo. Rio de Janeiro: COP; 1987. 
5. Lazarus RS, Launier S. Stress related transaction between person and environment. In: Dervin LA, Lewis M. Perspectives in international psychology. New York: Plenum; 1978. p. 287-327.

6. Menzies IEP. Nurses under stress. Int Nurs Rev. 1960;7(6): 9-16.

7. Bauk DA. Stress. Rev Bras Saúde Ocup. 1985;13(50):28-36.

8. Takahashi EIU. A emoção na prática de enfermagem: relatos de enfermeiros de UTI e UI [tese]. São Paulo: Escola de Enfermagem, Universidade de São Paulo; 1991.

9. Gentry WD, Parkes KR. Psychologic stress in intensive care unit and non-intensive care unit nursing: a review of the past decade. Heart Lung. 1982;11(1):43-7.

10. Corrêa A K. Sendo enfermeira no Centro de Terapia Intensiva. Rev Bras Enferm. 1995;48(3):233-4.

11. Brasil. M inistério da Saúde. DATASUS. Indicadores e dados básicos [texto na Internet]. Brasília; 2006. [citado 2006 set. 2]. Disponível em: http:/tabnet.datasus.gov. $\mathrm{br} / \mathrm{cgi} / \mathrm{idb2004/matriz.htm}$.

12. Spindola T, Santos RS. Mulher e trabalho: a história de vida de mães trabalhadoras de enfermagem. Rev Lat Am Enferm. 2003;11(5):593-600.
13. Stacciarini JMR, Tróccoli BT. O estresse na atividade ocupacional do enfermeiro. Rev Lat Am Enferm. 2001; 9(2):17-25.

14. Hays MA, All AC, Mannahan C, Cuaderes E, Wallace D. Reported stressors and ways of coping utilized by intensive care unit nurses. Dimens Crit Care Nurs. 2006; 25 (4):185-93.

15. Hudak CM, Gallo BM. Cuidados intensivos de enfermagem: uma abordagem holística. 6a ed. Rio de Janeiro: Guanabara-Koogan; 1997. Efeitos da UTI sobre o enfermeiro; p. 99-109.

16. M iyadahira AM K, Cruz DALM, Padilha KG, Kimura M, Sousa RMC. Recursos humanos das Unidades de Terapia Intensiva do município de São Paulo. Rev Lat Am Enferm. 1999;7(5):15-23.

17. Are new graduates hired in your unit? If not why not? What advice would you give them? Crit Care Nurse. 1993; 13(5):126-7.

18. Bianchi ERF. Enfermeiro hospitalar e o stress. Rev Esc Enferm USP. 2000;34(4):390-4.

\section{ANEXO}

\section{ESCALA BIANCHI DE STRESS (EBS)}

Este questionário tem a finalidade de levantar dados para conhecer a sua opinião quanto ao desempenho de suas atividades. NÃO PRECISA IDENTIFICAÇÃO. Assinale a alternativa que revele a sua percepção, levando em consideração os números:

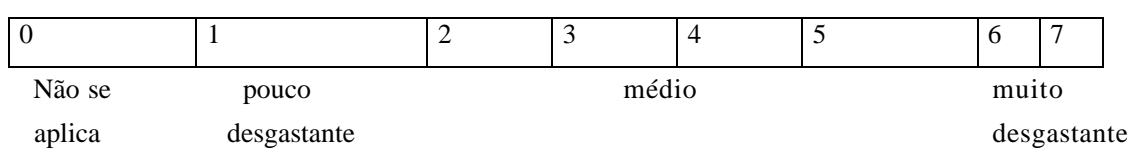

\section{PARTE 1}

Sexo: ( ) feminino $\quad$ ( ) masculino

Faixa etária: ( ) 20 a 30 anos
( ) 31 a 40 anos
( ) 41 a 50 anos
( ) mais de 50 anos

Cargo: 
continuação...

Unidade a que pertence:

Curso de pós-graduação: ( ) não

$$
\text { ( ) } \operatorname{sim} \quad \text { Qual (is) }
$$

Tempo de formado: ( ) menos de 1 ano

$$
\begin{aligned}
& \text { ( ) de } 2 \text { a } 5 \text { anos } \\
& \text { ( ) de } 6 \text { a } 10 \text { anos } \\
& \text { ( ) de } 11 \text { a } 15 \text { anos } \\
& \text { ( ) mais de } 16 \text { anos }
\end{aligned}
$$

\section{PARTE 2}

1. Previsão de material a ser usado

01234567

2. Reposição de material

01234567

3. Controle de material usado

01234567

4. Controle de equipamento

01234567

5. Solicitação de revisão e conserto de equipamentos

01234567

6. Levantamento de quantidade de material existente na unidade

01234567

7. Controlar a equipe de enfermagem

01234567

8. Realizar a distribuição de funcionários

9. Supervisionar as atividades da equipe

10. Controlar a qualidade do cuidado

11. Coordenar as atividades

12. Realizar o treinamento

13. Avaliar o desempenho dos funcionários

14. Elaborar escala mensal de funcionários

15. Elaborar relatório mensal da unidade

16. Admitir o paciente na unidade

17. Fazer exame físico do paciente

18. Prescrever cuidados de enfermagem

19. Avaliar as condições do paciente

20. Atender as necessidades do paciente

21. Atender as necessidades dos familiares

22. Orientar o paciente para o auto-cuidado

23. Orientar os familiares para cuidar do paciente

24. Supervisionar o cuidado de enfermagem prestado

25. Orientar para a alta do paciente

26. Prestar os cuidados de enfermagem

27. Atender às emergências na unidade

28. Atender aos familiares de pacientes críticos
01234567

01234567

01234567

01234567

01234567

01234567

01234567

01234567

01234567

01234567

01234567

01234567

01234567

01234567

01234567

01234567

01234567

01234567

01234567

01234567
01234567

continua... 
32. Realizar discussão de casos com a equipe multiprofissional

33. Participar de reuniões do Departamento de Enfermagem

01234567

34. Participar de comissão na instituição

01234567

35. Participar de eventos científicos

01234567

36. O ambiente físico da unidade

01234567

37. Nível de barulho da unidade

01234567

38. Elaborar rotinas, normas e procedimentos

01234567

39. Atualizar rotinas, normas e procedimentos

01234567

40. Relacionamento com outras unidades

01234567

41. Relacionamento com centro cirúrgico

01234567

42. Relacionamento com centro de materiais

01234567

43. Relacionamento com almoxarifado

01234567

44. Relacionamento com farmácia

01234567

45. Relacionamento com manutenção

01234567

46. Relacionamento com admissão/ alta de paciente

01234567

47. Definição das funções do enfermeiro

01234567

48. Realizar atividades burocráticas

01234567

49. Realizar atividades com tempo mínimo disponível

01234567

50. Comunicação com supervisores de enfermagem

01234567

51. Comunicação com administração superior

Sugestões e comentários: 